\title{
Glioblastoma multiforme outcomes of 107 patients treated in two Singapore institutions
}

\author{
Song Tao Timothy $\underline{\mathrm{Cheo}}^{1}$, MBBS, FRANzCR, Gek Hsiang $\underline{\operatorname{Lim}}^{2}$, BSc(Hons), MSc, Keith Hsiu Chin $\underline{\operatorname{Lim}}^{1,3}$, MBBS, FRANZCR
}

INTRODUCTION Glioblastoma multiforme (GBM) is the most common primary brain tumour in adults. Although the survival rate for GBM has improved with recent advancements in treatment, the prognosis remains generally poor. METHODS We conducted a retrospective review of GBM patients seen in National University Hospital, Singapore, and Tan Tock Seng Hospital, Singapore, from January 2002 to December 2011. Data on disease and treatment factors was collected and correlated with survival.

RESULTS Data on a total of 107 GBM patients was analysed. Their median survival time was 15.1 months and the two-year survival rate was $23.5 \%$, which is comparable with data published in other series. The factors associated with improved median survival time were radiotherapy dose $>50$ Gy (16.1 months vs. 8.7 months, $p=0.01)$ and adjuvant concurrent chemotherapy (16.4 months vs. 9.2 months, $p=0.003$ ).

CONCLUSION GBM confers a poor prognosis. Adjuvant radiotherapy and chemotherapy are associated with improved survival. Ethnicity may be a contributing factor to differences in GBM incidence and prognosis.

Keywords: brain tumour, clinical outcomes, glioblastoma multiforme

\section{INTRODUCTION}

High-grade gliomas (HGGs) are the most common type of primary brain tumour in adults. The majority of HGGs are of the glioblastoma multiforme (GBM) histological subtype. Although progress has been made in terms of improving the survival rate for GBM, most patients eventually relapse and succumb to the disease. The current treatment paradigm for GBM is multimodal, consisting of surgical resection as the mainstay, with postoperative radiation and chemotherapy. Better local control, achieved mainly through the addition of chemotherapy, and improvements in surgical and radiotherapy techniques have led to improvements in survival in recent years. ${ }^{(1,2)}$

Prognostication of GBM has traditionally been based on clinical factors such as age, functional status, extent of treatment received and histopathological factors (e.g. World Health Organization subtype). These factors have been well characterised in Caucasian populations, but have yet to be validated in our Asian population. There is also evidence to suggest that the molecular basis for HGGs and GBMs may differ among ethnic groups, ${ }^{(3)}$ and that a distinct pathway may exist in Asians. ${ }^{(4,5)}$ In this study, we retrospectively reviewed the clinical and demographic features of Asian patients with GBMs to establish the impact of these features on the prognosis of the disease.

\section{METHODS}

Ethical approval for this study was obtained from the Domain Specific Review Board, National Healthcare Group, Singapore. We reviewed the medical records of patients who were seen at the radiation oncology departments of two local tertiary hospitals
(National University Hospital and Tan Tock Seng Hospital) and were diagnosed with GBM during a ten-year period from January 2002 to December 2011. Patients were only included in the study if they had a histological diagnosis of GBM, either from a biopsy or excision. Non-residents who did not return for follow-up after initial resection were excluded.

Demographic data (i.e. age, gender and ethnicity), clinicopathological data (i.e. neurological function, mental status, performance status and duration of symptoms) and treatment parameters (i.e. extent of surgical resection, dose of radiotherapy and use of chemotherapy) were obtained from electronic or physical medical records. Data on the extent of surgical resection was reported by the surgeon and documented in the operating notes. Radiotherapy dose refers to the actual, rather than the initially planned, dose delivered to the tumour; this information was obtained from the electronic radiotherapy records. Data on patient deaths was obtained from the National Registry of Diseases Office, Health Promotion Board, Singapore. Survival was defined as the period beginning from the date of definitive histological diagnosis to the date of death.

Summary statistics were used to describe the demographic and clinical profiles of the patients. The Kaplan-Meier approach was used to estimate the probability of survival of the patients based on specific demographic factors. Cox regression was used to test for the effect of the various risk factors on survival. Univariate and multivariate analyses of the factors were done. A p-value $<0.05$ was considered to be statistically significant. All analyses were performed using STATA version 10.0 (StataCorp LP, TX, USA).

\footnotetext{
${ }^{1}$ Department of Radiation Oncology, National Cancer Institute Singapore, National University Health System, ${ }^{2}$ National Registry of Diseases Office, Health Promotion Board, ${ }^{3}$ NUS Yong Loo Lin School of Medicine, National University of Singapore, Singapore

Correspondence: Dr Timothy Cheo Song Tao, Associate Consultant, Department of Radiation Oncology, National Cancer Institute Singapore, National University Health System, 5 Lower Kent Ridge Road, Singapore 119074. Timothy_cheo@nuhs.edu.sg
} 
Table I. Median survival and two-year survival rate of the patients with glioblastoma multiforme $(\mathrm{n}=107)$, according to their demographic and clinical features.

\begin{tabular}{|c|c|c|c|c|c|}
\hline \multirow[t]{2}{*}{ Variable } & \multirow[t]{2}{*}{ No. (\%) } & \multirow[t]{2}{*}{ Median survival (mth) } & \multirow[t]{2}{*}{ 2-year survival rate (\%) } & \multicolumn{2}{|c|}{ p-value } \\
\hline & & & & Univariate & Multivariate \\
\hline Overall & $107(100.0)$ & 15.1 & 23.5 & - & - \\
\hline Age (yr) & & & & 0.35 & 0.07 \\
\hline$\leq 30$ & $8(7.5)$ & 15.1 & 50.0 & & \\
\hline $31-40$ & $7(6.5)$ & 12.2 & 42.9 & & \\
\hline $51-60$ & $35(32.7)$ & 16.3 & 22.9 & & \\
\hline $61-70$ & $24(22.4)$ & 11.9 & 19.5 & & \\
\hline$>70$ & $17(15.9)$ & 9.3 & 13.7 & & \\
\hline Ethnicity & & & & 0.16 & 0.24 \\
\hline Chinese & $82(76.6)$ & 14.9 & 19.3 & & \\
\hline Malay & $10(9.3)$ & 13.7 & 50.0 & & \\
\hline Indian & $8(7.5)$ & 7.1 & 25.0 & & \\
\hline Others & $7(6.5)$ & 22.3 & 27.8 & & \\
\hline Gender & & & & 0.44 & 0.96 \\
\hline Male & $65(60.7)$ & 13.7 & 22.1 & & \\
\hline Female & $42(39.3)$ & 16.3 & 25.6 & & \\
\hline Surgical resection & & & & 0.21 & 0.74 \\
\hline Excision & $90(84.1)$ & 15.8 & 25.8 & & \\
\hline Biopsy & $17(15.9)$ & 9.3 & 11.8 & & \\
\hline Radiotherapy dose (Gy) & & & & 0.01 & 0.94 \\
\hline$>50$ & $85(79.4)$ & 16.1 & 27.3 & & \\
\hline$\leq 50$ & $22(20.6)$ & 8.7 & 9.1 & & \\
\hline Temozolomide chemotherapy* & & & & 0.003 & 0.03 \\
\hline
\end{tabular}

*Data of 16 patients whose temozolomide chemotherapy status could not be determined from medical records and 1 patient who received non-temozolomide chemotherapy was excluded from analysis.

\section{RESULTS}

The data of 107 GBM patients was analysed in this study. They had a median survival of 15.1 months and two-year survival rate of $23.5 \%$ (Table I). The age range was $13-85$ years. GBM occurred most commonly in the sixth decade of life (32.7\%) and only $14.0 \%$ occurred in patients aged $<40$ years. Median survival, which was found to decrease with increasing age, was the worst in the $>70$ years age group (9.3 months).

Ethnic Malays appeared to be underrepresented in the study. Although the Malays comprise $15.0 \%$ of the general population in Singapore according to national statistics, they made up only $9.3 \%$ of the GBM patients in this study. ${ }^{(6)}$ The two-year survival rate was $50.0 \%$ among the Malay patients, as compared to $19.3 \%$ among the Chinese patients. However, this difference was not statistically significant $(p=0.16)$, given the small sample size. Male patients, who made up $60.7 \%$ of the 107 GBM patients, had a median survival of 13.7 months and twoyear survival rate of $22.1 \%$. In comparison, the female patients had a median survival of 16.3 months and a two-year survival rate of $25.6 \%$. Again, these differences were not statistically significant $(p=0.44)$.

Although most of the patients who underwent surgery had excisions $(84.1 \%)$ rather than biopsies $(15.9 \%)$, the association with survival was not significant $(\mathrm{p}=0.21)$. Among the patients who underwent excision, $16.7 \%$ underwent gross total resection and $21.1 \%$ underwent subtotal excision; the extent of excision was not reported in the remaining patients $(62.2 \%)$. Highdose adjuvant radiotherapy (defined as > 50 Gy), which was administered to $79.4 \%$ of the patients, was found to be associated with improved survival on univariate analysis $(p=0.01)$. The median survival and two-year survival rate were 16.1 months and $27.3 \%$, respectively, for the patients who received highdose radiotherapy, and 8.7 months and $9.1 \%$, respectively, for the patients who received $\leq 50$ Gy of radiotherapy. However, the association between high-dose adjuvant radiotherapy and survival was not sustained on multivariate analysis $(p=0.94)$. The probability of survival of the patients, according to the radiotherapy dose they received (i.e. > 50 Gy vs. $\leq 50$ Gy), is shown in Fig. 1.

Most of the patients $(68.9 \%)$ received chemotherapy in the form of oral temozolomide, which was given concurrently with radiotherapy and adjuvantly thereafter. Adjuvant concurrent chemotherapy with temozolomide was found to be associated with improved survival on both univariate and multivariate analyses $(p=0.003$ and $p=0.03$, respectively). The median survival and two-year survival rate was higher among the patients 


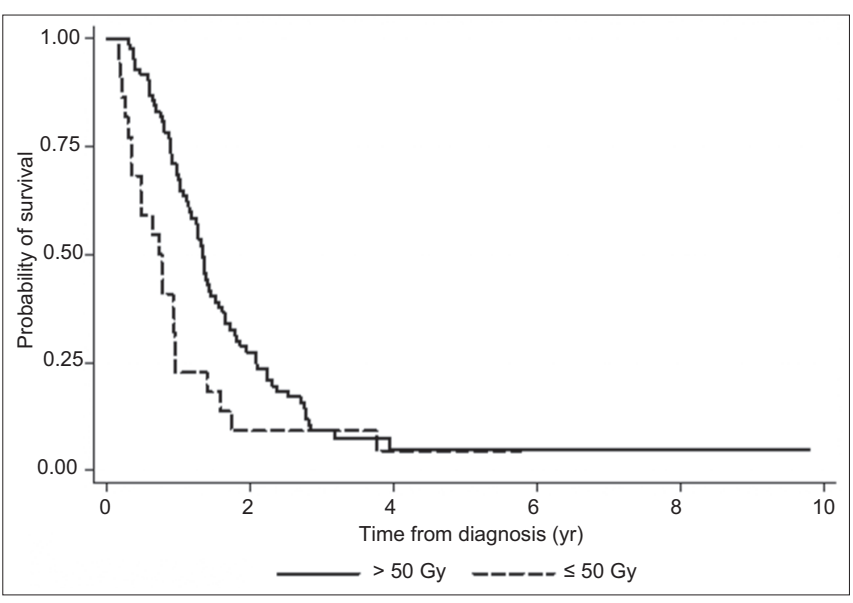

Fig. 1 Graph shows the overall survival of the patients, according to the radiotherapy dose they received.

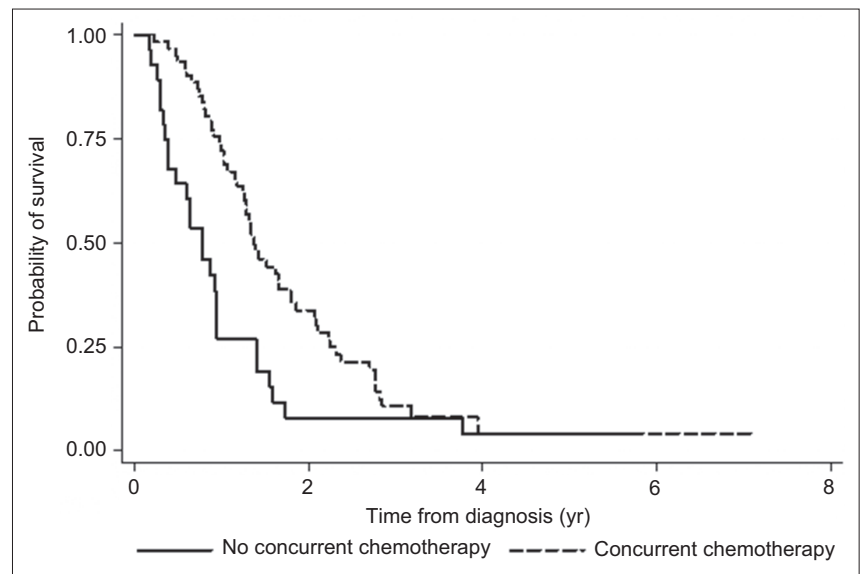

Fig. 2 Graph shows the overall survival of the patients, according to whether they underwent adjuvant concurrent chemotherapy treatment.

Table II. Survival outcomes of patients with GBM in population-based studies.(2,13,15-19)

\begin{tabular}{|c|c|c|c|}
\hline Population & Study period & Median survival (mth) & 2-year survival (\%) \\
\hline Canadian ${ }^{(15)}$ & $1982-1994$ & 7 & 11 \\
\hline Mexican ${ }^{(16)}$ & 1987-1994 & 16 & 20 \\
\hline Chinese $^{(13)}$ & 1999-2004 & 12 & 17 \\
\hline Korean ${ }^{(2)}$ & 1999-2004 & 11 & 20 \\
\hline Italian ${ }^{(17)}$ & $2002-2007$ & 9 & 25 \\
\hline French $^{(18)}$ & 2004-2006 & 9 & 12 \\
\hline Scottish(19) & 2004-2009 & 15 & 19 \\
\hline Singaporean (present study) & 2002-2011 & 15 & 24 \\
\hline
\end{tabular}

who had adjuvant concurrent chemotherapy than among the patients who did not (16.4 months and 33.7\% vs. 9.2 months and $7.7 \%$, respectively) (Table I). The probability of survival of the patients, according to whether they received adjuvant concurrent chemotherapy, is shown in Fig. 2.

\section{DISCUSSION}

The present study on the epidemiology of GBM in Singapore produced findings that closely resemble the results of international studies on GBM. The incidence of GBM peaked in the sixth decade of life $(32.7 \%)$ in our study, a trend that was similarly observed in the international studies. ${ }^{(1,2,7)}$ Our finding of a male predominance in the incidence of GBM (60.7\%) was also consistent with international studies, which reported a male predominance that ranged from $58 \%$ to $69 \% .^{(1,7,8)}$

Most of the patients in the present study underwent multimodal treatment, which has become the standard of care since the publication of Stupp et al's seminal paper in 2005. ${ }^{(9)}$ Although the results of our study suggest that there may be ethnic variations in the incidence and outcomes of GBM, the limited sample size did not permit us to demonstrate this conclusively. Nonetheless, such geoethnic variations are well described, such as in a number of population-based studies showing that the incidence of GBM is higher among Caucasians than other ethnic groups..$^{(7,10-12)}$ However, there has been no conclusive evidence that the clinical outcomes of GBM differ among ethnic groups. ${ }^{(13)}$ Population-based studies conducted in Korea and China showed that the survival of Asian patients was similar to that of Western populations. ${ }^{(2,13)}$ In one study that involved a racially diverse group of patients in America, there appeared to be improved survival among Asians as compared to other races. ${ }^{(14)}$ However, the authors hypothesised that the observed improved survival may be due to socioeconomic factors and access to care rather than intrinsic or genetic factors. ${ }^{(14)}$ In Singapore, where subsidised healthcare is highly accessible, it is likely that any observed ethnic difference in GBM outcome is due to intrinsic rather than extrinsic factors.

The survival outcomes in the present study are similar to those reported by other population-based studies that were conducted during the chemotherapy era (Table II). (2,13,15-19) Earlier studies reporting outcomes during the pre-temozolomide era tend to show poorer survival outcomes when compared to recent studies that were conducted during the chemotherapy era. While our study includes the period before and after data from Stupp's seminal trial was published in 2005, most of our patients had received adjuvant concurrent temozolomide chemotherapy, which likely accounted for their better survival outcome.

Multiple large-scale, retrospective studies have identified several clinicopathological factors as predictors of GBM outcome. ${ }^{(1,11,20)}$ These factors include age, performance status, histology, extent of surgical resection, and the addition of radiotherapy and chemotherapy as adjuvants. Some authors have attempted to stratify patients into prognostic groups based on these factors in order to predict survival. ${ }^{(21,22)}$ Despite the presence of such predictive models and calculators, ${ }^{(22)}$ differences 
in local populations and environments mean that these models and calculators need to be validated locally before they can be employed. (19) The present study has shown that the prognostic factors in our local population are similar to those reported worldwide. $^{(11,13,18,21)}$

Increased understanding of the molecular mechanisms of GBM has led to the development of predictive models that incorporate the use of molecular markers, which in turn has impacted the clinical outcomes of patients with GBM. One significant example is the development of an online GBM calculator by the European Organisation for Research and Treatment of Cancer. ${ }^{(23)}$ This calculator enables its users to predict survival data based on clinicopathological risk factors, such as $\mathrm{O}^{6}$-alkylguanine DNA alkyltransferase (MGMT) status. ${ }^{(23)}$ Besides MGMT, other genes that may be involved in the molecular pathogenesis of GBM are p52, PTEN, EGFR and $I D H 1{ }^{(24,25)}$ Variations in the incidence and outcomes of GBM among different ethnic groups may be due to differences in the molecular mechanisms of the disease. Several studies have shown differential rates of known mutations and the presence of entirely novel mutations in different ethnic groups. ${ }^{(3,26)}$ Studies on ethnic Malays have identified putative molecular mechanisms involving PTEN and loss of heterozygosity, which may be able to account for differences in the incidence and outcomes of GBM. ${ }^{(5)}$

The findings of the present study are consistent with the existing knowledge that adjuvant radiotherapy with the addition of temozolomide-based chemotherapy improves survival in patients with GBM. While the extent of surgical resection has been shown to impact survival in several large-scale studies, ${ }^{(27,28)}$ this association has not been reproduced in other studies. ${ }^{(29)}$ This may be, in part, due to differences in the terminology used to report the extent of resection. In the present study, surgeonreported resection extents were used and most of the surgeons did not categorically report the resection extents as gross total or subtotal. A more reliable approach, based on the evaluation of pre- and postoperative images to establish the extent of resection may well show the expected association.

To the best of our knowledge, this is the largest retrospective review of GBM in Singapore. The demographics of Singapore have seen a shift from a mainly resident populace to one with a significant migrant working population. This may alter the future incidence of GBM and delivery of care for GBM. Future studies on GBM in Singapore should be expanded to examine population-based data to verify whether there are ethnic variations in the incidence and prognosis of GBM, and to identify possible molecular mechanisms to account for the variations, should they exist. To this end, it would be beneficial to establish a nationwide tumour tissue bank to help advance future research and eventually develop individualised therapy for GBM.

To conclude, although GBM confers a grim prognosis, adjuvant chemotherapy and radiotherapy are associated with improved survival. An expansion of the present study to include population-based statistics may validate ethnic differences in the incidence and outcomes of GBM.

\section{REFERENCES}

1. Darefsky AS, King JT Jr, Dubrow R. Adult glioblastoma multiforme survival in the temozolomide era: a population-based analysis of Surveillance, Epidemiology, and End Results registries. Cancer 2012; 118:2163-72.

2. Jung KW, Yoo $\mathrm{H}$, Kong $\mathrm{HJ}$, et al. Population-based survival data for brain tumors in Korea. J Neurooncol 2012; 109:301-7.

3. Chen P, Aldape K, Wiencke JK, et al. Ethnicity delineates different genetic pathways in malignant glioma. Cancer Res 2001; 61:3949-54.

4. Das A, Tan WL, Teo J, Smith DR. Glioblastoma multiforme in an Asian population: evidence for a distinct genetic pathway. J Neurooncol 2002; 60:117-25.

5. Zainuddin N, Jaafar H, Isa MN, Abdullah JM. Malignant glioma: the involvement of loss of allelic heterozygosity and PTEN mutations in a group of Malay patients. Southeast Asian J Trop Med Public Health 2005; 36:748-56.

6. National Population and Talent Division, Singapore. Population in Brief 2013. Available at: http://www.nptd.gov.sg/portals/0/news/population-inbrief-2013.pdf. Accessed February 18, 2016.

7. Sadetzki S, Zach L, Chetrit A, et al. Epidemiology of gliomas in Israel: a nationwide study. Neuroepidemiology 2008; 31:264-9.

8. Kaneko S, Nomura K, Yoshimura T, Yamaguchi N. Trend of brain tumor incidence by histological subtypes in Japan: estimation from the Brain Tumor Registry of Japan, 1973-1993. J Neurooncol 2002; 60:61-9.

9. Stupp R, Mason WP, van den Bent MJ, et al; European Organisation for Research and Treatment of Cancer Brain Tumor and Radiotherapy Groups; National Cancer Institute of Canada Clinical Trials Group. Radiotherapy plus concomitant and adjuvant temozolomide for glioblastoma. New Engl J Med 2005; 352:987-96.

10. Darefsky AS, Dubrow R. International variation in the incidence of adult primary malignant neoplasms of the brain and central nervous system. Cancer Causes Control 2009; 20:1593-604.

11. Thumma SR, Fairbanks RK, Lamoreaux WT, et al. Effect of pretreatment clinical factors on overall survival in glioblastoma multiforme: a Surveillance Epidemiology and End Results (SEER) population analysis. World J Surg Oncol 2012; 10:75.

12. Robertson JT, Gunter BC, Somes GW. Racial differences in the incidence of gliomas: a retrospective study from Memphis, Tennessee. Br J Neurosurg 2002; 16:562-6.

13. Ma X, Lv Y, Liu J, et al. Survival analysis of 205 patients with glioblastoma multiforme: clinical characteristics, treatment and prognosis in China. J Clin Neurosci 2009; 16:1595-8.

14. Barnholtz-Sloan JS, Maldonado JL, Williams VL, et al. Racial/ethnic differences in survival among elderly patients with a primary glioblastoma. J Neurooncol 2007; 85:171-80.

15. Paszat L, Laperriere N, Groome $P$, et al. A population-based study of glioblastoma multiforme. Int J Radiat Oncol Biol Phys 2001; 51:100-7.

16. Lopez-Gonzalez MA, Sotelo J. Brain tumors in Mexico: characteristics and prognosis of glioblastoma. Surg Neurol 2000; 53:157-62.

17. Scoccianti S, Magrini SM, Ricardi $U$, et al. Patterns of care and survival in a retrospective analysis of 1059 patients with glioblastoma multiforme treated between 2002 and 2007: a multicenter study by the Central Nervous System Study Group of Airo (italian Association of Radiation Oncology). Neurosurgery 2010; 67:446-58.

18. Bauchet L, Mathieu-Daudé $H$, Fabbro-Peray $P$, et al; Société Française de Neurochirurgie (SFNC); Club de Neuro-Oncologie of the Société Française de Neurochirurgie (CNO-SFNC); Société Française de Neuropathologie (SFNP); Association des Neuro-Oncologues d'Expression Française (ANOCEF). Oncological patterns of care and outcome for 952 patients with newly diagnosed glioblastoma in 2004. Neuro Oncol 2010; 12:725-35.

19. Teo M, Clark B, MacKinnon M, et al. The validity of EORTC GBM prognostic calculator on survival of GBM patients in the West of Scotland. Br J Neurosurg 2014; 28:356-62.

20. Hulshof MC, Koot RW, Schimmel EC, et al. Prognostic factors in glioblastoma multiforme. 10 years experience of a single institution. Strahlenther Onkol 2001; 177:283-90.

21. Curran WJ Jr, Scott CB, Horton J, et al. Recursive partitioning analysis of prognostic factors in three Radiation Therapy Oncology Group malignant glioma trials. J Natl Cancer Inst 1993; 85:704-10.

22. Mirimanoff RO, Gorlia T, Mason W, et al. Radiotherapy and temozolomide for newly diagnosed glioblastoma: recursive partitioning analysis of the EORTC 26981/22981-NCIC CE3 phase III randomized trial. J Clin Oncol 2006; 24:2563-9.

23. European Organisation for Research and Treatment of Cancer. Nomograms 
for predicting survival of GBM patients. Available at: https://www.eortc be/tools/gbmcalculator/model1.aspx. Accessed February 18, 2016.

24. Ohgaki H, Dessen P, Jourde B, et al. Genetic pathways to glioblastoma: a population-based study. Cancer Res 2004; 64:6892-9.

25. Fukushima T, Favereaux A, Huang $\mathrm{H}$, et al. Genetic alterations in primary glioblastomas in Japan. J Neuropathol Exp Neurol 2006; 65:12-8.

26. Tunca B, Bekar A, Cecener G, et al. Impact of novel PTEN mutations in Turkish patients with glioblastoma multiforme. J Neurooncol 2007 82:263-9.
27. Simpson JR, Horton J, Scott C, et al. Influence of location and extent of surgical resection on survival of patients with glioblastoma multiforme: results of three consecutive Radiation Therapy Oncology Group (RTOG) clinical trials. Int J Radiat Oncol Biol Phys 1993; 26:239-44.

28. Lacroix M, Abi-Said D, Fourney DR, et al. A multivariate analysis of 416 patients with glioblastoma multiforme: prognosis, extent of resection, and survival. J Neurosurg 2001; 95:190-8.

29. Hess KR. Extent of resection as a prognostic variable in the treatment of gliomas. J Neurooncol 1999; 42:227-31. 